\title{
Mass Spectrometry-Based Quantification of Pseudouridine in RNA
}

\author{
Balasubrahmanyam Addepalli, Patrick A. Limbach
}

Department of Chemistry, Rieveschl Laboratories for Mass Spectrometry, University of Cincinnati, P.O. Box 210172, Cincinnati, OH 45221-0172, USA

\begin{abstract}
Direct detection of pseudouridine $(\psi)$, an isomer of uridine, in RNA is challenging. The most popular method requires chemical derivatization using $\mathrm{N}$-cyclohexyl-N'- $\beta$-(4-methylmorpholinum ethyl) carbodiimide $p$-tosylate $(\mathrm{CMCT})$ followed by radiolabeled primer extension mediated by reverse transcriptase. More recently, mass spectrometry (MS)-based approaches for sequence placement of pseudouridine in RNA have been developed. Nearly all of these approaches, however, only yield qualitative information regarding the presence or absence of pseudouridine in a given RNA population. Here, we have extended a previously developed liquid chromatography tandem mass spectrometry (LC-MS/MS) method to enable both the qualitative and quantitative analysis of pseudouridine. Quantitative selected reaction monitoring (SRM) assays were developed using synthetic oligonucleotides, with or without pseudouridine, and the results yielded a linear relationship between the ion abundance of the pseudouridine-specific fragment ion and the amount of pseudouridine-containing oligonucleotide present in the original sample. Using this quantitative SRM assay, the extent of pseudouridine hypomodification in the conserved T-loop of tRNA isolated from two different Escherichia coli strains was established.
\end{abstract}

Key words: Pseudouridine quantification, tRNA, Mass spectrometry, Selected reaction monitoring, SRM, Detection of RNA modifications, Oligonucleotides

\section{Introduction}

$\mathrm{P}$ seudouridine $(\psi)$ is the most prevalent post-transcriptional modification in ribonucleic acids (RNA) [1, 2]. This isomer of uridine is commonly found in non-coding RNAs, including transfer RNA (tRNA), ribosomal RNA (rRNA), small nuclear RNA (snRNA), and small nucleolar RNA (snoRNA). Moreover, pseudouridine is found in all phylogenetic domains of life (Archaea, Eubacteria, and Eukarya) [3]. Although a precise molecular role for this

Electronic supplementary material The online version of this article (doi:10.1007/s13361-011-0137-5) contains supplementary material, which is available to authorized users.

Correspondence to: Patrick A. Limbach; e-mail: Pat.Limbach@uc.edu modification is unclear, pseudouridine has been shown to confer rigidity and stabilize RNA structure, enhance local base stacking, and is involved in imino proton- $\mathrm{H}_{2} \mathrm{O}$ coordination [4, 5]. Pseudouridine modulates codon-anticodon interactions between messenger RNA and tRNA [6] and facilitates ribosome assembly [7].

Pseudouridine is formed by site-specific isomerization at the polyribonucleotide level following recognition of the target uridine in the context of the RNA sequence or structure of the site of interest by a group of enzymes called pseudouridine synthases $[8,9]$. Studies on prokaryotes and eukaryotes have shown that deletion of pseudouridine synthase genes has a negative impact on cell growth and protein synthesis rates $[10,11]$ indicating the critical function of this modification in the translational apparatus. However, identifying the exact location of pseudouridine in the context of its parent RNA is key to an improved 
understanding of the function of this posttranscriptional modification.

Pseudouridine is a mass-silent modification, therefore it does not exhibit a mass shift compared with its unmodified counterpart upon post-transcriptional formation in RNA. The most widely used method for detection and sequence placement of pseudouridine is based on $\psi$-specific irreversible derivatization using the reagent $N$-cyclohexyl- $N-\beta-(4-$ methylmorpholinium) ethylcarbodiimide $p$-tosylate (CMCT) followed by reverse transcriptase mediated extension of radiolabeled primer and subsequent separation on a denaturing polyacrylamide gel [12]. Although effective, this method has some drawbacks and limitations, which have spurred development of mass spectrometry-based approaches for the detection and sequence placement of pseudouridine in RNA [13]. The two major mass spectrometry-based approaches involve enzymatic digestion of RNA to either nucleosides or oligonucleotides, yielding samples suitable for analysis.

The presence of pseudouridine can be detected by total enzymatic hydrolysis of RNA into nucleosides and subsequent analysis by liquid chromatography/electrospray ionization mass spectrometry (LC-ESI-MS) [14, 15]. The sensitivity and specificity of pseudouridine detection in a RNA nucleoside mixture was further enhanced upon derivatization with methyl vinyl sulfone [15]. A limitation of this approach is the lack of information relating to the sequence location of pseudouridine in the RNA.

To determine the specific sequence placement of this modification, RNase mapping was employed in combination with chemical derivatization [16-18]. RNase mapping uses base-specific endoribonucleases that generate a mixture of oligoribonucleotides, which are amenable to accurate mass measurements by ESI or MALDI (matrixassisted laser desorption/ionization) mass spectrometry. As pseudouridine is a mass silent modification, the RNA (before or after enzymatic treatment) is chemically derivatized with either CMCT $[16,17]$ or acrylonitrile (cyanoethylation) [18] to covalently link a mass tag to pseudouridine that enables differentiation of pseudouridine from uridine by mass spectrometry. Identification of pseudouridine oligonucleotides is accomplished by mass spectrometric comparison of untreated and CMCT or acrylonitrile treated samples. Mass differences of $252 \mathrm{Da}$ (CMCT-treated) or $53 \mathrm{Da}$ (acrylonitrile) between the two samples are used to identify RNase digestion products that contain pseudouridine [16-18].

A more recent development is a tandem mass spectrometry (MS/MS) approach compatible with LC-MS based RNase mapping of nucleic acids [19]. This approach was developed to take advantage of the unique but stable $\mathrm{C}-\mathrm{C}$ glycosidic bond, which contrasts with the typically labile $\mathrm{C}-$ $\mathrm{N}$ glycosidic bond in uridine or other nucleosides. The stable $\mathrm{C}-\mathrm{C}$ glycosidic bond yields unique fragmentation pathways during collision-induced dissociation (CID) leading to the production of pseudouridine-specific fragment ions. These diagnostic ions include the doubly dehydrated nucleoside anion $m / z 207$ and its MS/MS product ion at $m / z 164$ as well as the fragmentation product at $m / z 165$ arising from MS/MS of $\mathrm{m} / \mathrm{z} 225$. Selective detection of these fragmentation pathways enabled the identification of pseudouridine in a given oligonucleotide by selected reaction monitoring (SRM). Specifically, pseudouridines can be identified by scanning the SRM transition of $\mathrm{m} / \mathrm{z} 207$ to 164. Alternatively, the SRM transition of $m / z 225$ to 165 can be used to selectively identify the presence of a 5 ' terminal pseudouridine in the RNase digestion product [19]. McCloskey and co-workers used these SRM assays and chemical derivatization to identify pseudouridines in $23 \mathrm{~S}$ rRNAs of Haloarcula marismortui and Deinococcus radiodurans [20], and the $16 \mathrm{~S}$ rRNA of Thermus thermophilus [21].

While effective, the approaches described above can only yield qualitative information about the presence or absence of pseudouridine in a given RNA. Most often, the modification at a given position may not be present at stoichiometric amounts as the degree or level of modification may be modulated for regulatory purposes like growth or stress response [22, 23]. Several methods have, therefore, been developed to quantify pseudouridine in RNA. In one, RNase $\mathrm{H}$ mediated cleavage of RNA, ${ }^{32} \mathrm{P}$ substitution of phosphate backbone, thin layer chromatography (TLC) of hydrolyzed nucleotides followed by phosphorimaging allows for the quantification of this post-transcriptional modification [24]. A ligation-based method was developed that allows recognition of the modification site and quantification on denaturing PAGE [25]. Although these methods are highly specific, in that modification at a single sequence location can be quantified, the overall assay is not readily applied to multiple modifications. Beyond the LC-MS analysis of nucleoside digests mentioned above, which can be performed quantitatively [26, 27], a novel method enabling the relative quantification of pseudouridine from the base hydrolysis of oligoribonucleotides has recently appeared [28]. Here we have developed a quantitative assay for the routine sequence-specific analysis of pseudouridine. This SRM-based assay utilizes the pseudouridine-specific transitions identified by McCloskey and coworkers [19] to enable one to determine the degree of pseudouridine modification in RNA via RNase mass mapping and LCMS/MS, where the oligonucleotides are less than $20 \mathrm{nt}$. Further, we have applied this quantitative assay to document the variability of pseudouridine hypomodification in the conserved T- loop of E. coli tRNAs.

\section{Experimental}

\section{Materials}

Oligoribonucleotides were obtained from Dharmacon RNAi Technologies (Lafayette, CO, USA) and deprotected as per the manufacturer's instructions. Escherichia coli strain K12 was purchased from American Type Culture Collection 
(ATCC, Manassas, VA, USA), and E. coli Rosetta 2(DE3) strain was purchased from Novagen (Gibbstown, NJ, USA). 1,1,1,3,3,3-Hexafluoroisopropanol (HFIP), ammonium acetate, Tri-Reagent, lysozyme chloride from chicken egg white, chloroform, 2-propanol, absolute ethanol, 1,2-diaminocyclohexyltetraacetic acid monohydrate (CDTA), and $E$. coli $\mathrm{tRNA}_{\mathrm{QUA}}^{\text {Tyr }}$ were used as received from Sigma-Aldrich (St. Louis, MO). HPLC-MS grade methanol, water and acetonitrile were obtained from Honeywell Burdick \& Jackson, Inc. (Muskegon, MI, USA). Triethylamine (TEA), sodium chloride, sodium acetate, sodium hydroxide, and sodium dodecyl sufate (SDS) were purchased from Fisher Scientific (Fairlawn, NJ, USA). Molecular biology grade Tris base (tris(hydroxymethyl)aminomethane), and trishydrochloride were purchased from Promega (Madison, WI, USA). Bacto yeast extract and bacto tryptone were used as received from BD Biosciences (Sparks, MD, USA). Sodium citrate was purchased from Mallinckrodt Baker, Inc. (Paris, KY, USA). UltraPure agarose, bacterial alkaline phosphatase (BAP), and Dyna beads M-280 were purchased from Invitrogen Corporation (Carlsbad, CA, USA). RNase T1 was purchased from Roche Molecular Biochemicals (Indianapolis, IN, USA). Sep-Pak C18 cartridges were obtained from Waters (Milford, MA, USA). The 5'-biotin labeled oligodeoxynucleotide 5'biotin-GGCAGAGGTCT CAGGTTCGAATCCTGTCGGGCGCG-3' was obtained from Integrated DNA Technologies, Inc. (Coralville, IA, USA).

\section{Isolation of E. coli $t R N A$ s}

E. coli $\mathrm{K} 12$ cells were cultured with regular Luria-Bertani (LB) medium, while the medium was supplemented with chloramphenicol $(25 \mu \mathrm{g} / \mathrm{mL})$ for culturing Rosetta 2(DE3) cells as per manufacturer's instructions. The cells were harvested at the end of log phase $\left(0.8-0.9 \mathrm{OD}_{600}\right)$ and either used straightaway or frozen at $-80{ }^{\circ} \mathrm{C}$ until further use. The tRNAs were isolated using Tri-Reagent as described [29].

\section{Purification of tRNA Arg (CCG)}

Rosetta 2(DE3) cells (Novagen) are designed to alleviate codon bias by complementing the insufficient tRNA pools in the parental cell line K12 through overexpression of rare tRNAs by a multicopy plasmid. One of the overexpressed tRNAs is tRNA $\mathrm{Arg}$. This tRNA was affinity purified by using a biotinylated oligodeoxynucleotide whose sequence is complementary to the $3^{\prime}$-end of $\mathrm{tRNA}_{\mathrm{CCG}}^{\mathrm{Arg}}$. The biotinylated oligodeoxynucleotide was coupled to streptavidincoated magnetic beads (M-280, Invitrogen) following the manufacturer's instructions. Hybridization of the TriReagent isolated tRNAs with the magnetic beads was performed at $70{ }^{\circ} \mathrm{C}$ for 10 min followed by washing with stirring at $37^{\circ} \mathrm{C}$ for $2 \mathrm{~h}$. The affinity purified tRNA $\mathrm{CCG}_{\mathrm{Arg}}^{\mathrm{Arg}}$ was eluted using $15 \mathrm{mM}$ sodium chloride, $1.7 \mathrm{mM}$ sodium citrate, and a $0.1 \%$ SDS solution.

\section{Enzymatic Digestion of tRNAs}

Guanosine specific ribonuclease RNase T1 was precipitated and purified as described [30]. For digestion, 500 units of RNase T1 and 0.0005 units BAP were added to $10 \mu \mathrm{g}$ of tRNA and $5 \mu \mathrm{L}$ of $220 \mathrm{mM}$ ammonium acetate buffer. The reaction mixture was incubated in a $37{ }^{\circ} \mathrm{C}$ water bath for $2 \mathrm{~h}$.

\section{Ribonuclease Digestion Products}

tRNA sequences were obtained from the Transfer RNA Database [31]. Sequences were theoretically digested using Mongo Oligo Mass Calculator (ver. 2.06; http://library.med. utah.edu/masspec/).

\section{Mass Spectrometry}

Direct sample infusion (ESI-MS) and LC-MS/MS experiments were performed on a Thermo Scientific (Waltham, MA, USA) LTQ XL linear ion trap mass spectrometer fitted with an electrospray ionization (ESI) probe at a fixed vertical spray angle $\left(60^{\circ}\right)$ in the Ion Max Source. The electrospray ion source was coupled to a Finnegan Surveyor MS Pump Plus HPLC system and Finnegan Micro AS autosampler for automated injections.

For direct infusion, synthetic oligoribonucleotide samples, combined with CDTA $(0.01 \mathrm{nmol})$ and TEA $(0.0015 \%)$, were dissolved to achieve a final concentration of $10 \mathrm{pmol} / \mu \mathrm{L}$ in 50:50 water/methanol. Samples were infused via a syringe pump at a flow rate of $3 \mu \mathrm{L} / \mathrm{min}$ and the spectra were acquired in negative ion mode. The settings for pseudouridine detection were: $275^{\circ} \mathrm{C}$ ESI source; $4.5 \mathrm{kV}$ spray voltage; sheath, auxiliary and sweep gas to 25,14 , and 10 arbitrary units; ions were tuned for $m / z 207$ to enable automatic adjustment of capillary, tube lens, and other ion optics voltages. This tune file was used for detection and quantification of all the $\psi$-specific transitions for all the tested oligonucleotides.

For LC-MS/MS analysis, reverse phase ion-pairing chromatography was performed on an Xbridge MS C18 $1.0 \times 150 \mathrm{~mm}$ column, $3.5 \mu \mathrm{m}$ particle size and $135 \AA$ pore size (Waters, Milford, MA, USA) at a flow rate of $40 \mu \mathrm{L} /$ min. Mobile phase A consisted of $16.3 \mathrm{mM}$ TEA/400 mM HFIP at pH 7.0 in water; equal amounts of mobile phase A and methanol were combined to produce mobile phase B. The mobile phase gradient starting at $5 \% \mathrm{~B}$ increased to 10 $\% \mathrm{~B}$ at $2 \mathrm{~min}$, and $95 \% \mathrm{~B}$ at $52 \mathrm{~min}$. The mobile phase composition was held at $95 \% \mathrm{~B}$ for 5 min prior to reequilibrating the column for $15 \mathrm{~min}$ under initial mobile phase conditions before the next injection. The entire column effluent was coupled to the electrospray probe via PEEK tubing (1/16 in o.d. 0.005 in i.d., Upchurch Scientific). Negative ion mass spectra were recorded in continuum mode from $\mathrm{m} / \mathrm{z} 450$ to 2000 in scan event 1 to acquire the $\mathrm{m} / \mathrm{z}$ values of intact oligonucleotides. To identify 
the ion charge states, data were acquired in enhanced scan mode. For detecting pseudouridine diagnostic ions, two additional scan events were used with an elevated source voltage of $90 \mathrm{~V}$ for in-source fragmentation. The presence of $\psi$ in the dissociated ions was detected by two consecutive scan events using a reaction monitoring function that recorded the transition from $\mathrm{m} / \mathrm{z} 207$ to 164 for internal pseudouridines (scan event 2) and the transition from $\mathrm{m} / \mathrm{z}$ 225 to 165 for 5 ' terminal $\psi$ (scan event 3 ) at a normalized collision energy of $30 \%$. Vendor supplied Xcalibur software was used for all data acquisition and processing.

\section{Quantitative Analysis}

To evaluate the suitability of this method to quantitate unknowns, a set of standard solutions with varied but known concentrations of $\psi$-containing oligonucleotides were subjected to LC-MS analysis. Matching the retention time of a specific $m / z$ value of an oligonucleotide ion with the $\psi$-specific transition occurring at the same time indicates the presence of pseudouridine containing oligonucleotide in the eluent. SRM peak area values were used to generate calibration curves in Microsoft Excel from which the pseudouridine concentrations of the sample of interest could be determined.

\section{Results and Discussion}

\section{Identification of Pseudouridine-specific Fragment Ions in Oligonucleotides}

The SRM approach for detecting $\psi$-diagnostic fragment ions was originally developed on a triple quadrupole instrument [19]. To confirm that an ion trap mass spectrometer also generates identical SRM transitions for pseudouridine detection, the oligoribonucleotide $\mathrm{A} \psi \mathrm{CAG}$ (monoisotopic mass $_{\text {calculated }}$ 1552.26) was studied. This oligonucleotide upon in-source dissociation was expected to yield $\mathrm{m} / \mathrm{z} 207$, and selection of this precursor and further ion trap fragmentation should yield the diagnostic ion at $\mathrm{m} / \mathrm{z}$ 164. The A $\psi \mathrm{CAG}$ oligonucleotide exhibited predominantly a deprotonated doubly charged ion at $\mathrm{m} / \mathrm{z} 775.25$ (Figure 1a). In-source fragmentation followed by selection and fragmentation of $\mathrm{m} / \mathrm{z}$ 207 in the ion trap yielded an abundant product ion at $\mathrm{m} / \mathrm{z} 164$ (Figure 1b). The negative control, AUCAG, was subjected to the same experimental conditions (Figure 1c) with no product ion at $\mathrm{m} / \mathrm{z} 164$ detected (Figure 1d). These data demonstrate that the $m / z 207$ to 164 SRM transition can be observed with an ion trap mass spectrometer, and this transition is specific to pseudouridine-containing oligoribonucleotides as reported previously [19]. Next, we sought to establish parameters by

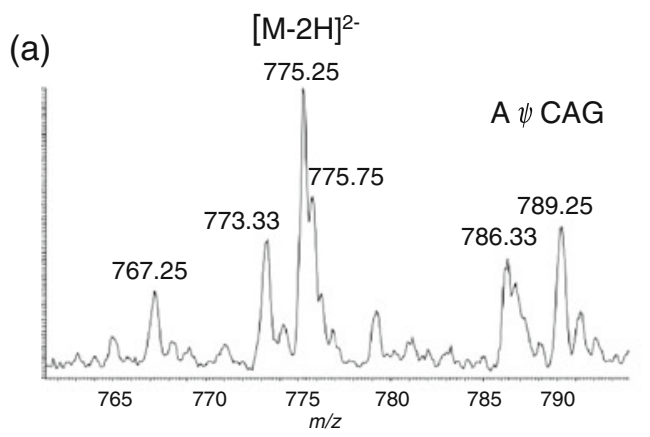

(b)

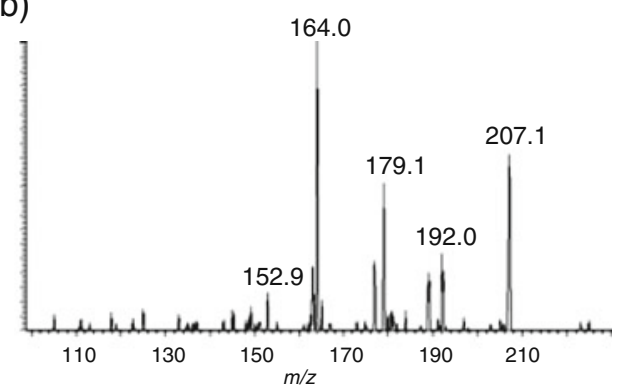

(c)

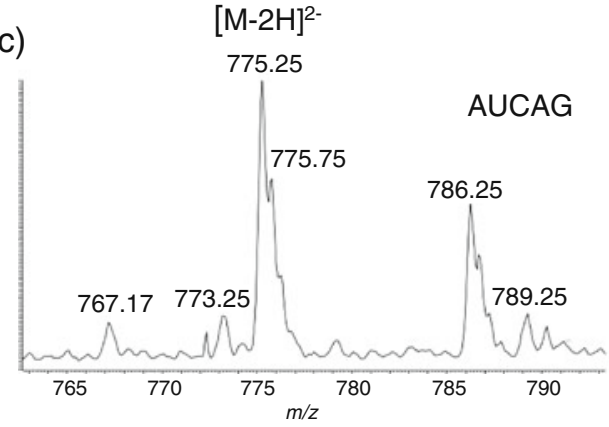

(d)

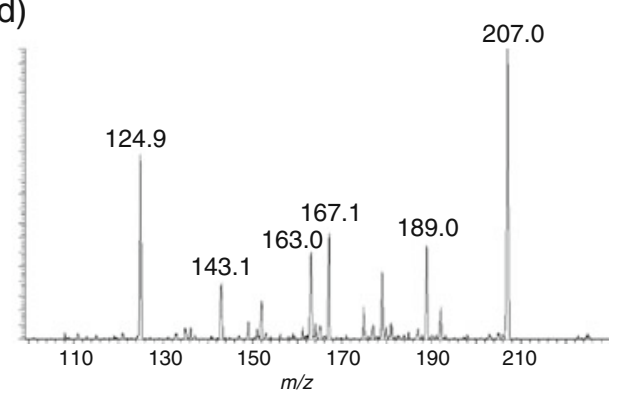

Figure 1. (a) ESI-MS of a $10 \mathrm{pmol} / \mu \mathrm{L}$ solution of $A \psi C A G$. (b) $E S I-M S / M S$ of a $10 \mathrm{pmol} / \mu \mathrm{L}$ solution of $A \psi C A G$. In-source fragmentation (90 V source lens) was applied to dissociate A $\psi C A G$. The precursor ion $(\mathrm{m} / \mathrm{z} 207)$ was then selected for collisioninduced dissociation (CID) yielding the mass spectrum seen. The pseudouridine-specific fragment ion at $\mathrm{m} / \mathrm{z} 164$ is readily detected. (c) ESI-MS of a $10 \mathrm{pmol} / \mathrm{\mu L}$ solution of AUCAG. (d) ESI-MS/MS of a $10 \mathrm{pmol} / \mathrm{\mu L}$ solution of AUCAG. In-source fragmentation (90 V source lens) was applied to dissociate AUCAG. The precursor ion $(\mathrm{m} / z 207)$ was then selected for collisioninduced dissociation (CID) yielding the mass spectrum seen. As expected, no pseudouridine-specific fragment ion at $\mathrm{m} / z 164$ can be detected 
which this SRM transition could be used to quantify pseudouridine within RNAs.

\section{Quantification Studies}

To establish whether a quantitative SRM assay could be developed for pseudouridine, pairs of synthetic oligonucleotides that share an identical sequence but differ for the presence or absence of pseudouridine at a defined site were used. In general, a set of standard solutions containing known amounts of a pseudouridine-containing oligonucleotide was mixed with the same sequence lacking pseudouridine where the total concentration of the two oligonucleotides does not exceed 70 pmol. The samples consisted of $0,5,10,20,35,50,60,70 \mathrm{pmol}$ of a pseudouridine-containing oligonucleotide and 70, 65, 60, $50,35,20,10,0$ pmol of a pseudouridine-lacking oligonucleotide. These samples were injected onto a C-18 column and analyzed by LC-MS/MS.

An example of the process used to generate quantitative SRM assays is illustrated using the oligonucleotide $\mathrm{G}(\psi / \mathrm{U})$ G. Injection of an equimolar mixture of the two oligonucleotides yielded two partially resolved chromatographic peaks, with singly charged ions $(\mathrm{m} / \mathrm{z}$ 933.08) being more abundant than doubly charged ions $(m / z$ 466.16) (Supplemental Figure S1). Monitoring the pseudouridine specific SRM transition of $\mathrm{m} / \mathrm{z} 207$ to 164 identified the earlier eluting peak as the pseudouridine-containing oligonucleotide. An SRM calibration curve for $\mathrm{G} \psi \mathrm{G}$ was generated using the peak area for the $\mathrm{m} / \mathrm{z} 207$ to 164 transition. Three injections of each mixture were analyzed. As seen in Figure 2a, a linear response $\left(\mathrm{R}^{2}=0.998\right)$ was obtained over the concentration range of interest ( 0 to $60 \mathrm{pmol})$.

Similar results were obtained from other oligonucleotides. The T $\psi C G$ :TUCG $\left(\mathrm{R}^{2}=0.975\right)$ and A $\psi$ CAG:AUCAG $\left(\mathrm{R}^{2}=\right.$ 0.991) mixtures yielded linear responses (Figure 2b, c) along with chromatographic resolution of the two components (Supplemental Figures S2 and S3). Differences in assay sensitivity were found among these three pairs of oligonucleotides with G $\psi \mathrm{G}$ being the most sensitive and T $\psi \mathrm{CG}$ the least sensitive, although the differences among these shorter oligonucleotides were less than an order of magnitude.

Next we sought to determine whether the SRM quantification assay could be extended to larger oligonucleotides as well as oligonucleotides containing more than one pseudouridine. An 11-mer sequence containing two internal uridines, UAACUAUGACG, was used along with the single and double pseudouridine analogs UAAC $\psi$ AUGACG and UAAC $\psi A \psi$ GACG. For these larger oligonucleotides, the pseudouridine containing analogs were not separated from the 11-mer containing only uridines (Supplemental Figure S4). During LC-MS, the doubly charged ions $(\mathrm{m} / \mathrm{z}$ 1735.6) were consistently of higher abundance than the triply charged anions $(\mathrm{m} / \mathrm{z} 1156.8)$.

While both pseudouridine analogs yielded linear calibration curves (Figure $2 \mathrm{~d}$, e), quantification was only possible up to $60 \mathrm{pmol}$. At $70 \mathrm{pmol}$, the response began to saturate yielding a non-linear response (data not shown). Not unexpectedly, the sensitivity for UAAC $\psi$ AUGACG was reduced compared with the shorter oligonucleotides discussed above. However, the sensitivity essentially doubled between UAAC $\psi$ AUGACG and UAAC $\psi A$ AGACG, consistent with this SRM assay yielding a linear response to the amount of pseudouridine within a particular oligonucleotide. While the SRM assay response does appear to reflect the amount of pseudouridine within a particular oligonucleotide, it is noted that UAAC $\psi A \psi \mathrm{AACG}$ was less consistent, particularly at higher concentrations, than UAAC $\psi$ AUGACG or any of the smaller oligonucleotides. Moreover, because this method relies on peak area measurements from the mass spectral data, the precision will be less than methods where internal standards are used.

Having confirmed that internal pseudouridines can be quantified via an SRM assay, we next examined whether the $\mathrm{m} / \mathrm{z} 225$ to $165 \mathrm{SRM}$ transition, which is unique to terminal pseudouridines, could also be used in a quantitative fashion. The first oligonucleotide pair investigated was $\psi \mathrm{GG}$ and UGG. As developed by McCloskey and co-workers, terminal pseudouridines yield the unique $\mathrm{m} / \mathrm{z} 225$ to 165 SRM transition, while all pseudouridine-containing oligonucleotides generate the $m / z 207$ to 164 SRM transition. As shown in Supplemental Figure S5, $\psi$ GG can be identified by either SRM transition. Comparing these transitions for use in quantitation of pseudouridine in $\psi \mathrm{GG}$, no significant differences between them were found (Figure 3a). Neither SRM transition yielded a sensitive response; in particular, quantification was not possible below 20 pmol of the pseudouridine containing oligonucleotide. Moreover, comparing the results from G $\psi \mathrm{G}$ with $\psi \mathrm{GG}$ (where the two oligonucleotides are isomers), the terminal pseudouridine results in a much less reproducible fragmentation, reducing the quality of the calibration curve $\left(\mathrm{G} \psi \mathrm{G} \mathrm{R}^{2}=0.998\right.$ versus $\psi \mathrm{GG} \mathrm{R} \mathrm{R}^{2}=$ 0.947). These results suggest quantification of small oligonucleotides with terminal pseudouridines may be less accurate and less sensitive than comparable oligonucleotides with an internal pseudouridine.

As before, a longer oligonucleotide was also analyzed. The same 11-mer as above, UAACUAUAACG, was used with $\Psi$ AACUAUAACG to enable comparisons with the internal pseudouridine SRM behavior. A mixture of the two 11-mers was not resolved on the C-18 column (Supplemental Figure S6), consistent with the chromatographic behavior of the internal pseudouridine analogs. However, while the sensitivity of the $\mathrm{m} / \mathrm{z} 225$ to 165 SRM transition for $\psi$ AACUAUAACG was less than the sensitivity of the $\mathrm{m} / \mathrm{z} 207$ to 164 SRM transition for UAAC $\psi A$ AUGACG, monitoring the terminal pseudouridine SRM yielded a linear response $\left(\mathrm{R}^{2}=0.991\right)$ with reasonable precision (Figure $3 b$ ). Thus, it appears that quantification of terminal pseudouridines by the specific SRM transition would be more appropriate for longer, rather than shorter, oligonucleotides. 
(a)

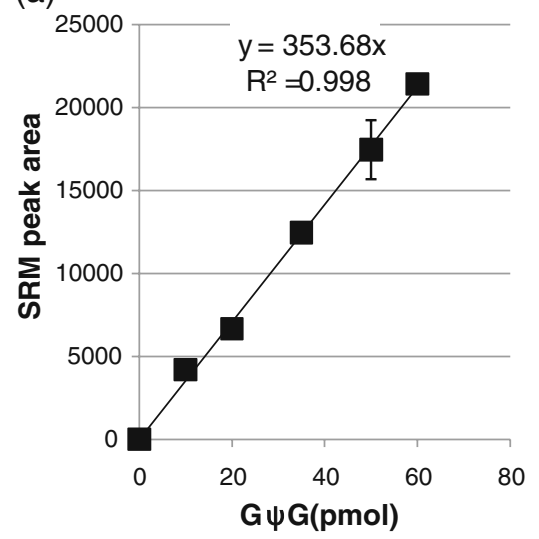

(c)

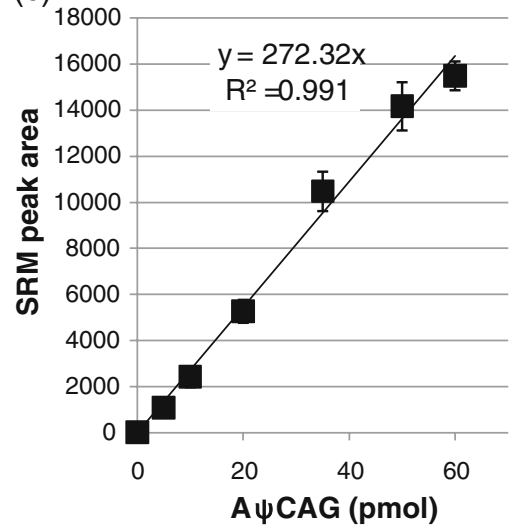

(e)

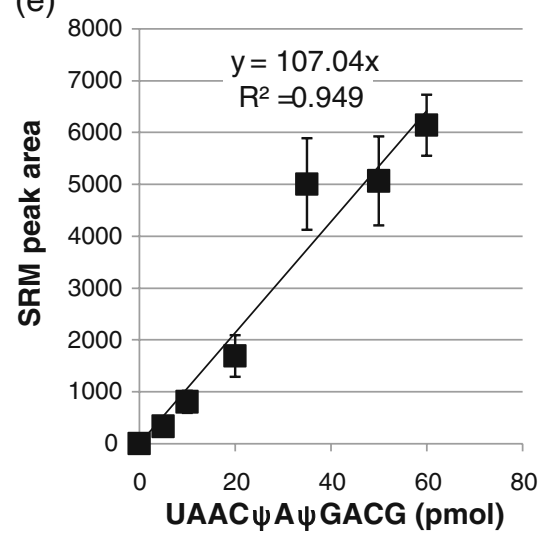

(b)

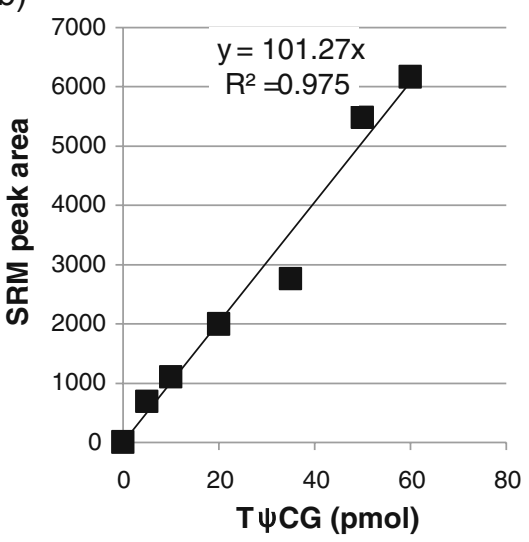

(d)

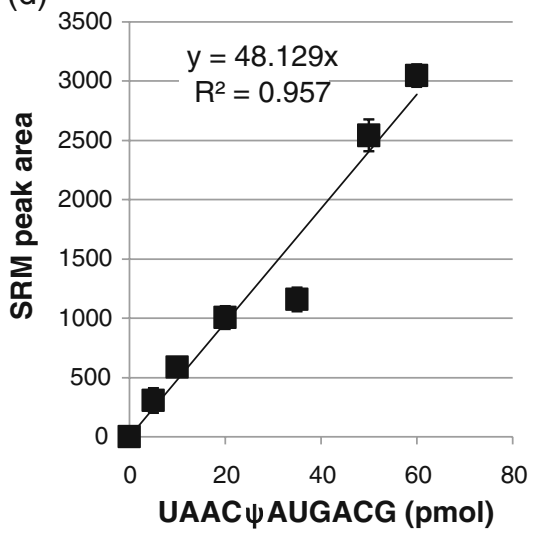

Figure 2. Calibration curves for the selected reaction monitoring (SRM) transition $m / z 207$ to 164 for (a) $G \psi G$, (b) $m^{5} U_{\psi} C G$, (c) A $\psi C A G$, (d) UAAC $\psi A$ AUGACG, and (e) UAAC $\psi A \psi G A C G$. Calibration curves were generated by LC-MS/MS analysis of defined amounts of each pseudouridine-containing oligonucleotide in combination with its uridine-containing analog, where the total oligonucleotide amount was $70 \mathrm{pmol}$. SRM transition peak areas, determined in triplicate, were plotted against the amount of pseudouridine-containing oligonucleotide and the resulting data fit to a linear least squares function. Although the sensitivities are sample dependent, all oligonucleotides yielded linear calibration curves with high precision

\section{Application of the SRM Assay for Quantification of Pseudouridine}

Having established that the pseudouridine specific SRM transitions $\mathrm{m} / \mathrm{z} 207$ to 164 and $\mathrm{m} / \mathrm{z} 225$ to 165 could be used to quantify pseudouridine in oligonucleotides, we next sought to apply this method for the quantification of pseudouridine in various biological samples. The samples investigated were Escherichia coli transfer RNAs.

Pseudouridine is one of the highly conserved and most common modifications in the 7-base T-loop of tRNAs. The T-loop has four strongly conserved bases, which include $\psi 55$, and two semi-conserved bases [22, 32]. Pseudouridine can be found less frequently at many other sites in tRNA in a 
(a)

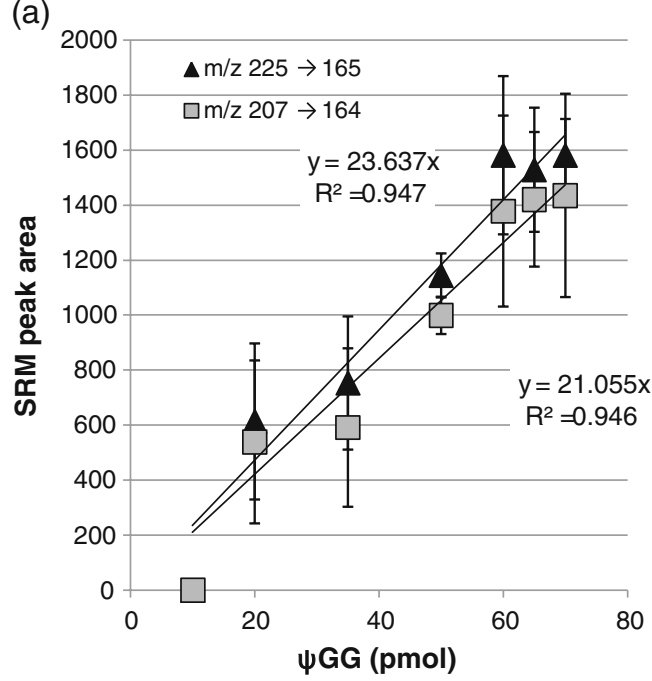

(b)

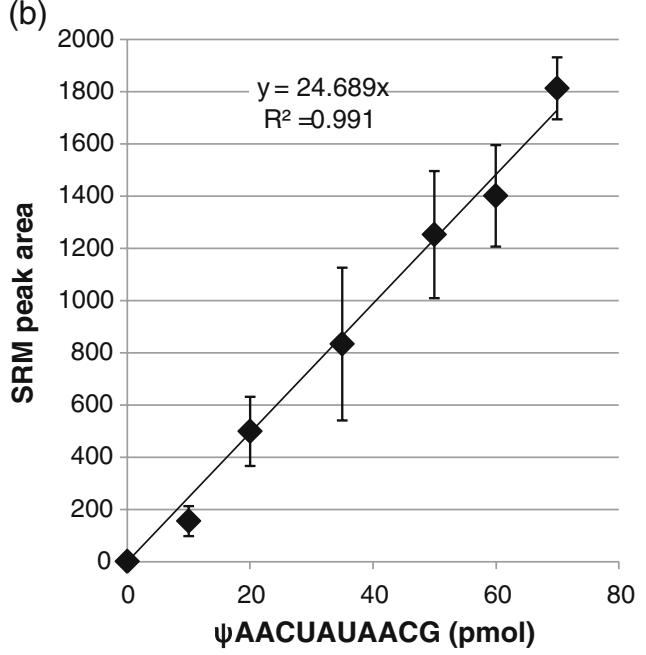

Figure 3. (a) Calibration curves for the selected reaction monitoring (SRM) transition $m / z 207$ to 164 (filled triangle) and the SRM transition $\mathrm{m} / \mathrm{z} 225$ to 165 (filled square) for $\psi \mathrm{GG}$. Both SRM transitions yielded similar results as evaluated by their sensitivity and reproducibility (linearity). (b) Calibration curve for the selected reaction monitoring (SRM) transition $\mathrm{m} /$ $z 225$ to 165 for $\psi$ AACUAUAACG. While the sensitivity of the SRM assay is similar to that found for the smaller $\psi \mathrm{GG}$ oligonucleotide, the 11-mer did yield improved linearity. Calibration curves were generated by LC-MS/MS analysis of defined amounts of each pseudouridine-containing oligonucleotide in combination with its uridine-containing analog, where the total oligonucleotide amount was 70 pmol. SRM transition peak areas, determined in triplicate, were plotted against the amount of pseudouridine-containing oligonucleotide and the resulting data fit to a linear least squares function

domain specific manner [33]. Nucleosides Gm18 in the conserved D loop and $\psi 55$ interact in the tRNA tertiary structure by hydrogen bonding, thereby stabilizing the L shape of tRNA [34]. $\mathrm{m}^{5} \mathrm{U} \psi \mathrm{CG}$ (where $\mathrm{m}^{5} \mathrm{U}=\mathrm{rT}$ ) is part of the conserved pentanucleotide $\mathrm{Gm}^{5} \mathrm{U} \psi \mathrm{CG}$ present in the T- loop of all E. coli tRNAs [22] except those tRNAs encoding isoleucine, leucine, and initiator tRNAs. Digestion of $E$. coli tRNAs containing the conserved $\mathrm{Gm}^{5} \mathrm{U} \psi \mathrm{CG}$ T-loop sequence with RNase $\mathrm{T} 1$ results in the tetranucleotide $\mathrm{m}^{5} \mathrm{U} \psi \mathrm{CGp}$. Initial studies on the nucleotide composition of this tetranucleotide indicated that both pseudouridine and uridine appeared to be present in the electrophoretically purified RNase T1 sample. These data suggested that uridine is not stoichiometrically modified to pseudouridine at this sequence location [22]. Thus, we decided to examine the extent of pseudouridine modification in E. coli tRNAs that yield the tetranucleotide $\mathrm{m}^{5} \mathrm{U} \psi \mathrm{CGp}$ using the SRM quantitative assay.

The SRM calibration curve for $\mathrm{m}^{5} \mathrm{U} \psi \mathrm{CG}$ generated previously (Figure 2b) was used for quantification of pseudouridine in the T-loop of E. coli tRNAs. A commercially available $E$. coli tRNA tRNA $\mathrm{QUA}_{\mathrm{QUA}}^{\mathrm{Tyr}}$ was analyzed. The sequence of $t R N A_{\mathrm{QUA}}^{\mathrm{Tyr}}$, including expected posttranscriptional modifications, is shown in Figure 4a. Digestion with RNase $\mathrm{T} 1$ and alkaline phosphatase will yield $\mathrm{m}^{5} \mathrm{U} \psi \mathrm{CG}$, which can then be quantitatively analyzed. Quantitative analysis was done by two separate SRM assay measurements, with the amount of starting material doubled between assays.

Results from LC-MS/MS analysis of $E$. coli $\mathrm{tRNA}_{\mathrm{QUA}}^{\mathrm{Tyr}}$ are presented in Figure 5. Figure $5 \mathrm{a}$ is the total ion chromatogram (TIC) from LC-MS analysis of the RNase $\mathrm{T} 1$ digest. Figure $5 \mathrm{~b}$ is the SRM assay for the $\mathrm{m} / \mathrm{z} 207$ to 164 transition. Three peaks were detected from the RNase T1 digest. The first peak arises from $\mathrm{m} / \mathrm{z} 606.2$ (Figure 5c), which is the doubly charged anion of $\mathrm{m}^{5} \mathrm{U} \psi \mathrm{CG}$. The second peak corresponds to an RNase T1 oligonucleotide from $E$. coli tRNA His $\left(\mathrm{m}^{2} \mathrm{~A} \psi \psi \mathrm{CCAG}\right)$ that has previously been found as a co-purified contaminant tRNA in commercial $E$. coli tRNA ${ }_{\mathrm{QUA}}^{\mathrm{Tyr}}$ samples [17]. The final peak arises from the RNase $\mathrm{T} 1$ digestion product $\mathrm{ACUQUAms} \mathrm{i}^{2} \mathrm{i} A \mathrm{~A} \psi \mathrm{CUG}(\mathrm{Q}=$ queuosine), which is found in the anticodon stem and loop region of $E$. coli $\mathrm{tRNA}_{\mathrm{QUA}}^{\mathrm{Tyr}}$. Quantitative analysis of two separate samples (1X and $2 \mathrm{X}$ ) yielded consistent results for the amount of pseudouridine in the T-loop of the commercial E. coli tRNA ${ }_{\mathrm{QUA}}^{\mathrm{Tyr}}$ sample (Table 1). Based on these

(a)

E. colitRNA Tyr (QUA)

$5^{\prime}$ - pGGUGGGG $\left[\mathrm{s}^{4} \mathrm{U}\right.$ ] UCCCGAGC [Gm] GCCAAAGGGAGCAG ACU [Q] UA [ms $\left.{ }^{2} i^{6} \mathrm{~A}\right]$ A [ $\psi$ ] CUGCCGUCAUCGACUUCGAAGG $\left[\mathrm{m}^{5} \mathrm{U}\right][\psi]$ ] CGAAUCCUUCCCCCACCACCA - 3 '

(b)

$$
\begin{aligned}
& \text { E. colitRNA Arg (CCG) } \\
& 5^{\prime} \text { - pGCGCCCGUAGCUCAGC [D] GGA [D] AGAGCGCUGCC } \\
& {\left[\mathrm{s}^{2} \mathrm{C}\right] \text { UCCG }\left[\mathrm{m}^{1} \mathrm{G}\right] \text { AGGCAGAG }\left[\mathrm{m}^{7} \mathrm{G}\right] \text { UCUCAGG }\left[\mathrm{m}^{5} \mathrm{U}\right][\psi \mathrm{H}] \mathrm{CG}} \\
& \text { AAUCCUGUCGGGCGCGCCA-3' }
\end{aligned}
$$

Figure 4. RNA sequence, including posttranscriptional modifications, for (a) E. coli tRNA $\mathrm{TUA}_{\mathrm{QUA}}^{\mathrm{Ty}}$ and (b) E. coli $\mathrm{tRNA}_{\mathrm{CCG}}^{\mathrm{Arg}}$ 


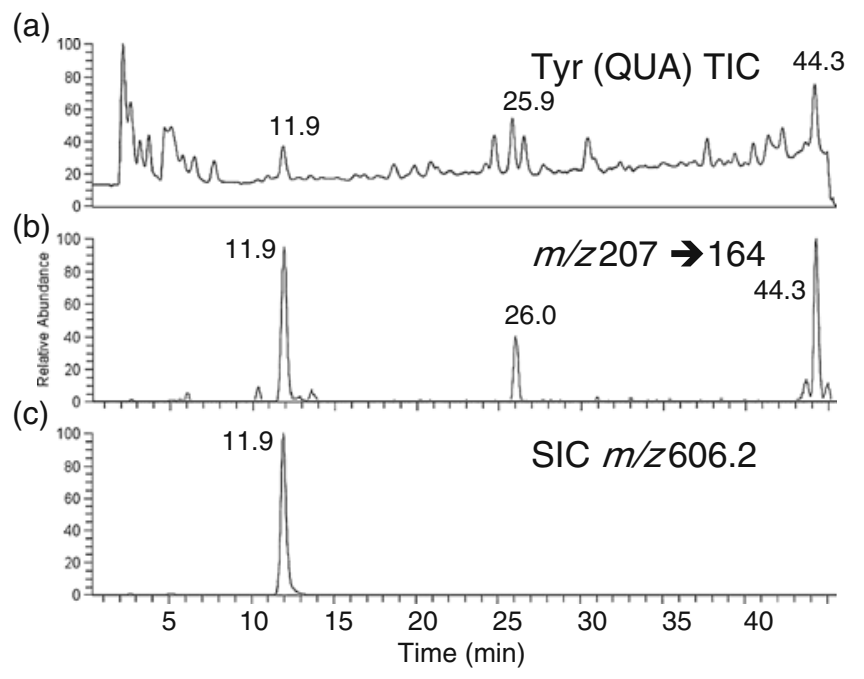

Figure 5. Quantitative analysis of pseudouridine in the Tloop tetramer $\mathrm{m}^{5} \mathrm{U} \psi \mathrm{CG}$ of $E$. coli $\mathrm{tRNA}_{\mathrm{QUA}}^{\mathrm{Tyr}}$. The tRNA was digested using RNase T1 and BAP and analyzed by LC-MS/ MS. Quantification of pseudouridine was performed using the SRM transition $\mathrm{m} / \mathrm{z} 207$ to 164 and the $\mathrm{m}^{5} \mathrm{U} \psi \mathrm{CG}$ calibration curve in Figure 2b. Results are listed in Table 1. (a) Total ion chromatogram (TIC) of LC-MS analysis of RNase T1 digest of tRNA $\mathrm{QUA}_{\mathrm{QU}}^{\mathrm{Ty}}$. (b) Pseudouridine-specific SRM transition. Three RNase T1/BAP digestion products contain pseudouridine (see text). (c) Selected ion chromatogram (SIC) of $m / z$ 606.2, which arises from the doubly charged anion of $\mathrm{m}^{5} \mathrm{U} \psi \mathrm{CG}$

analyses, pseudouridine is present $67 \%$ of the time in the Tloop, consistent with the prior report that uridine is not stoichiometrically modified to pseudouridine at this sequence location [22].

Hypomodified E. coli tRNAs can be generated when a tRNA gene is overexpressed by transforming cells with a plasmid containing one or more copies of the tRNA gene $[23,35]$. Previous studies on overexpression of $E$. coli tRNAs Leu [35] and Glu [23] both reported reduced levels of pseudouridinylation, although precise quantitative measurements and sequence-specific assignments of hypomodification were not discussed. Thus, quantitative analysis of pseudouridine levels in the T-loop of an overexpressed tRNA should reveal an even more significant level of (a)

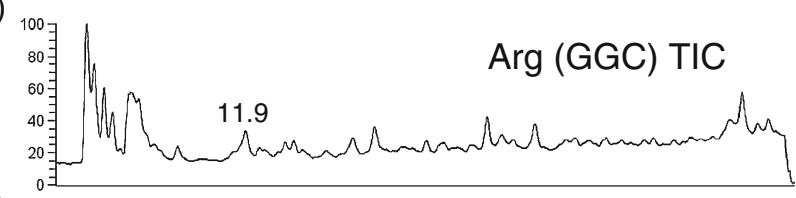

(b)

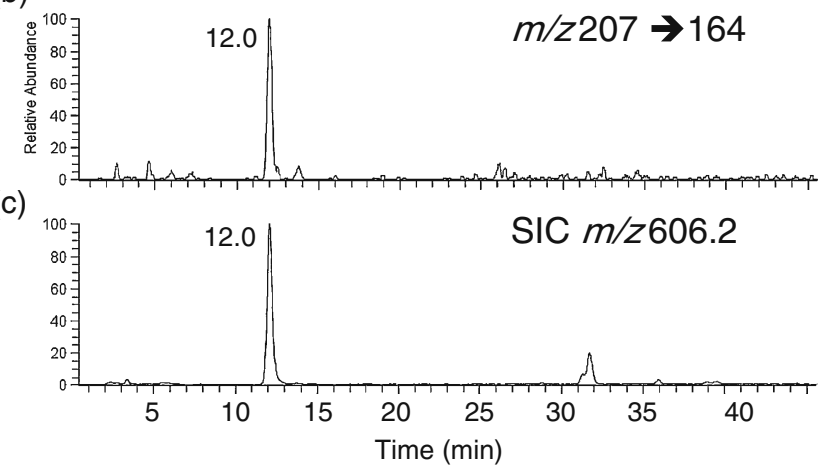

Figure 6. Quantitative analysis of pseudouridine in the Tloop tetramer $\mathrm{m}^{5} \mathrm{U} \psi \mathrm{CG}$ of $E$. coli $\mathrm{tRNA}_{\mathrm{CCG}}^{\mathrm{Arg}}$. The tRNA was digested using RNase T1 and BAP and analyzed by LC-MS/ MS. Quantification of pseudouridine was performed using the SRM transition $m / z 207$ to 164 and the $\mathrm{m}^{5} \mathrm{U} \psi \mathrm{CG}$ calibration curve in Figure 2b. Results are listed in Table 1. (a) Total ion chromatogram (TIC) of LC-MS analysis of RNase T1 digest of tRNA $\mathrm{Arg}$. (b) Pseudouridine-specific SRM transition. Only one RNase T1/BAP digestion product contains pseudouridine (see text). (c) Selected ion chromatogram (SIC) of $\mathrm{m} / \mathrm{z}$ 606.2, which arises from the doubly charged anion of $\mathrm{m}^{5} \mathrm{U} \psi \mathrm{CG}$

hypomodification than that found for the commercial E. coli tRNA. To test this possibility, E. coli tRNA Arg CCG $_{\text {as }}$ purified from a Rosetta2 strain, which contains the Arg (CCG) gene on a multicopy plasmid. The sequence of $\mathrm{tRNA}_{\mathrm{CCG}}^{\mathrm{Arg}}$, including expected post-transcriptional modifications, is shown in Figure 4b. Results from LC-MS/MS analysis of $E$. coli $\mathrm{tRNA} \mathrm{ACG}_{\mathrm{Arg}}$ are shown in Figure 6. Only a single peak for the $m / z 207$ to 164 SRM assay transition was detected in the RNase T1 digest of this tRNA (Figure 6b), consistent with the presence of only a single pseudouridine in the T-loop [31], which was confirmed by detection of the doubly charged anion for the tetranucleotide at $\mathrm{m} / \mathrm{z} 606.2$ (Figure 6c). Quantitative analysis of two separate samples (1X and $2 \mathrm{X})$ yielded consistent results for the amount of

Table 1. Quantitative Analysis of Pseudouridine in the T-loop tetramer $\mathrm{m}^{5} \mathrm{U} \psi \mathrm{CG}$ of Two E. coli tRNAs. The tRNAs were Digested using RNase T1 and BAP and Analyzed by LC-MS/MS. Quantification of Pseudouridine was Performed using the SRM Transition $m / z 207$ to 164 and the $\mathrm{m}^{5} \mathrm{U} \psi \mathrm{CG}$ Calibration Curve in Figure 2b. Mass Spectral Data are Found in Figures 5 and 6. Both tRNAs were Hypomodified, with the Overexpressed Significantly Less Modified at $\psi 55$ than the Normally Expressed tRNA $\mathrm{QUA}_{\mathrm{TU}}^{\mathrm{Ty}}$

\begin{tabular}{lccc}
\hline \multicolumn{1}{c}{ Sample } & Assay amount (pmol) & Experimentally observed (pmol) & Modifications/pmol \\
\hline $\mathrm{m}^{5} \mathrm{U} \psi \mathrm{CG} / \mathrm{m}^{5} \mathrm{UUCG}$ & $50 / 50 \mathrm{pmol}$ & $50 \pm 2.78$ & 1.00 \\
Tyr (QUA) & $29 \mathrm{pmol}$ & $20 \pm 1.56$ & 0.68 \\
& $14.2 \mathrm{pmol}$ & $9 \pm 1.3$ & 0.66 \\
$\operatorname{Arg}(\mathrm{CCG})$ & $32 \mathrm{pmol}$ & 9 & 0.28 \\
& $16 \mathrm{pmol}$ & 4 & 0.25 \\
\hline
\end{tabular}


pseudouridine in the T-loop of the overexpressed E. coli tRNA $\mathrm{CCG}$ sample (Table 1). In this case, only $25 \%$ of tRNA contained a pseudouridine posttranscriptional modification in place of uridine. These data support prior reports describing undermodification of tRNAs in overexpression systems, and, importantly, this SRM quantitative assay now allows for the sequence-specific characterization of pseudouridine levels in RNAs.

\section{Conclusions}

A quantitative mass spectrometry-based assay to characterize pseudouridine, the most prevalent post-transcriptional modification in RNA, has been developed. The amount of pseudouridine in a given isomeric oligonucleotide mixture can be determined even in a background of its uridine-containing analog via a pseudouridine-specific SRM assay. Both pseudouridine-specific SRM transitions ( $\mathrm{m} / \mathrm{z} 207$ to 164 and $\mathrm{m} / \mathrm{z} 225$ to 165 ) were developed into quantitative assays. The 5 '-terminus specific transition $(\mathrm{m} / \mathrm{z}$ 225 to 165 ) is less sensitive than the $\mathrm{m} / \mathrm{z} 207$ to 164 transition, although the sensitivity for the 5'-terminus pseudouridine does not appear to be affected by the length of the oligonucleotide. The sensitivity of the $m / z 207$ to 164 transition was, in general, found to be inversely dependent on the length of the oligonucleotide. The linear range for quantification was found to be 5 pmol to $60 \mathrm{pmol}$, although this range was more limited for oligonucleotides that contained multiple pseudouridines and for the 5'-terminus transition. Although these data demonstrate the site-specific quantification of pseudouridine by mass spectrometry, the method is dependent on the ability to generate a calibration curve for each pseudouridine-containing oligonucleotide of interest. While particular oligonucleotide sequences, especially RNase digestion products containing multiple post-transcriptional modifications, may not be available commercially, for those sequences where calibration standards can be obtained, this assay is easily amenable to automation for the quantitative analysis of pseudouridine in a specific sequence context from a large number of samples.

Using this assay, the levels of pseudouridine at $\psi 55$ in the conserved $\mathrm{m}^{5} \mathrm{U} \psi \mathrm{CG}$ tetramer present in the T-loop of E. coli tRNAs were determined for two isoaccepting tRNAs. Not surprisingly, $\psi 55$ levels were reduced from an overexpressed tRNA compared with that from a normally expressed tRNA. However, while earlier results suggested hypomodification of $\psi 55$ in E. coli tRNAs, this is the first time a quantitative measure of $\psi 55$ has been reported. This assay will enable additional studies into pseudouridine amounts at a sequencespecific level, which can be useful to further elucidate the functional role(s) of this post-transcriptional modification. Further, these results demonstrate that quantitative SRM assays for other RNA modifications are feasible, and additional efforts are on-going to extend quantitative SRM assays into methylation levels of RNAs as well as to use this existing assay to examine pseudouridine modification from larger RNAs, such as rRNAs.

\section{Acknowledgments}

The authors acknowledge financial support of this work by the National Institutes of Health (GM 58843) and the University of Cincinnati. The authors thank Thomas Riffelmacher for assistance with tRNA purification.

\section{References}

1. Limbach, P.A., Crain, P.F., McCloskey, J.A.: Summary: The modified nucleosides of RNA. Nucleic Acids Res 22, 2183-2196 (1994)

2. Rozenski, J., Crain, P.F., McCloskey, J.A.: The RNA Modification Database. Nucleic Acids Res 1999(27), 196-197 (1999)

3. Charette, M., Gray, M.W.: Pseudouridine in RNA: What, where, how, and why. IUBMB Life 49, 341-351 (2000)

4. Arnez, J.G., Steitz, T.A.: Crystal structure of unmodified tRNA(Gln) complexed with glutaminyl-tRNA synthetase and ATP suggests a possible role for pseudo-uridines in stabilization of RNA structure. Biochemistry 33, 7560-7567 (1994)

5. Davis, D.R.: Stabilization of RNA stacking by pseudouridine. Nucleic Acids Res 23, 5020-5026 (1995)

6. Davis, D.R., Veltri, C.A., Nielsen, L.: An RNA model for investigation of pseudouridine stabilization of the codon-anticodon interaction in tRNA(Lys), tRNA(His), and tRNA(Tyr). J Biomol Struct Dyn 15, 1121-1132 (1998)

7. Cunningham, P.R., Richard, R.B., Weitzmann, C.J., Nurse, K., Ofengand, J.: The absence of modified nucleotides affects both in vitro assembly and in vivo function of the $30 \mathrm{~S}$ ribosomal subunit of Escherichia coli. Biochimie 73, 789-796 (1991)

8. Gu, X., Liu, Y., Santi, D.V.: The mechanism of pseudouridine synthase I as deduced from its interaction with 5-fluorouracil-tRNA. Proc. Natl. Acad. Sci. U.S.A. 96, 14270-14275 (1999)

9. Spedaliere, C.J., Ginter, J.M., Johnston, M.V., Mueller, E.G.: The pseudouridine synthases: Revisiting a mechanism that seemed settled. $J$. Am. Chem. Soc. 126, 12758-12759 (2004)

10. Raychaudhuri, S., Conrad, J., Ofengand, J.: A pseudouridine synthase required for the formation of two universally conserved pseudouridines in ribosomal RNA is essential for normal growth of Escherichia coli. RNA 4, 1407-1417 (1998)

11. Lecointe, F., Simos, G., Sauer, A., Hurt, E.C., Motorin, Y., Grosjean, H.: Characterization of yeast protein Deg1 as pseudouridine synthase (Pus3) catalyzing the formation of C38 and C39 in tRNA anticodon loop. J. Biol. Chem. 273, 1316-1323 (1999)

12. Ofengand, J., Del Campo, M., Kaya, Y.: Mapping pseudouridines in RNA molecules. Methods 25, 365-373 (2001)

13. Durairaj, A., Limbach, P.A.: Mass spectrometry of the fifth nucleoside: A review of the identification of pseudouridine in nucleic acids. Anal. Chim. Acta 623, 117-125 (2008)

14. Pomerantz, S.C., McCloskey, J.A.: Analysis of RNA hydrolysates by liquid chromatography-mass spectrometry. Methods Enzymol 193, 796824 (1990)

15. Emmerechts, G., Herdewijn, P., Rozenski, J.: Pseudouridine detection improvement by derivatization with methyl vinyl sulfone and capillary HPLC-mass spectrometry. J. Chromatogr. B Anal. Technol. Biomed. Life Sci. 825, 233-238 (2005)

16. Patteson, K.G., Rodicio, L.P., Limbach, P.A.: Identification of the mass-silent post-transcriptionally modified nucleoside pseudouridine in RNA by matrix-assisted laser desorption/ionization mass spectrometry. Nucleic Acids Res. 29, E49 (2001)

17. Durairaj, A., Limbach, P.A.: Improving CMC-derivatization of pseudouridine in RNA for mass spectrometric detection. Anal. Chim. Acta 612, 173-181 (2008)

18. Mengel-Jorgensen, J., Kirpekar, F.: Detection of pseudouridine and other modifications in tRNA by cyanoethylation and MALDI mass spectrometry. Nucleic Acids Res. 30, e135 (2002)

19. Pomerantz, S.C., McCloskey, J.A.: Detection of the common RNA nucleoside pseudouridine in mixtures of oligonucleotides by mass spectrometry. Anal. Chem. 77, 4687-4697 (2005) 
20. Del Campo, M., Recinos, C., Yanez, G., Pomerantz, S.C., Guymon, R., Crain, P.F., McCloskey, J.A., Ofengand, J.: Number, position, and significance of the pseudouridines in the large subunit ribosomal RNA of Haloarcula marismortui and Deinococcus radiodurans. RNA 11, 210-219 (2005)

21. Guymon, R., Pomerantz, S.C., Crain, P.F., McCloskey, J.A.: Influence of phylogeny on posttranscriptional modification of rRNA in thermophilic prokaryotes: The complete modification map of $16 \mathrm{~S}$ rRNA of Thermus thermophilus. Biochemistry 45, 4888-4899 (2006)

22. Zamir, A., Holley, R.W., Marquisee, M.: Evidence for the occurrence of a common pentanucleotide sequence in the structures of transfer ribonucleic acids. J. Biol. Chem. 240, 1267-1273 (1965)

23. Madore, E., Florentz, C., Giegé, R., Sekine, S., Yokoyama, S., Lapointe, J.: Effect of modified nucleotides on Escherichia coli tRNA $^{\text {Glu }}$ structure and on its aminoacylation by glutamyl-tRNA synthetase. Predominant and distinct roles of the $\mathrm{mnm}^{5}$ and $\mathrm{s}^{2}$ modifications of U34. Eur J Biochem. 266, 1128-1135 (1999)

24. Zhao, X., Yu, Y.T.: Detection and quantitation of RNA base modifications. RNA 10, 996-1002 (2004)

25. Dai, Q., Fong, R., Saikia, M., Stephenson, D., Yu, Y.T., Pan, T., Piccirilli, J.A.: Identification of recognition residues for ligation-based detection and quantitation of pseudouridine and $\mathrm{N}^{6}$-methyladenosine. Nucleic Acids Res. 35, 6322-6329 (2007)

26. Chan, C.T.Y., Dyavaiah, M., DeMott, M.S., Taghizadeh, K., Dedon, P. C., Begley, T.J.: A Quantitative Systems Approach Reveals Dynamic Control of tRNA Modifications during Cellular Stress. PLoS Genet. 6, e1001247 (2010)
27. Brück1, T., Globisch, D., Wagner, M., Müller, M., Carell, T.: Parallel isotope-based quantification of modified tRNA nucleosides. Angew. Chem. Int. Ed. 48, 7932-7934 (2009)

28. Taucher, M.; Ganisl, B.; Breuker, K. Identification, localization, and relative quantitation of pseudouridine in RNA by tandem mass spectrometry of hydrolysis products. Int. J. Mass Spectrom. 2011, in press.

29. Lee, C., Kramer, G., Graham, D.E., Appling, D.R.: Yeast mitochondrial initiator tRNA is methylated at guanosine 37 by the Trm5-encoded tRNA (guanine-N1-)-methyltransferase. J. Biol. Chem. 282, 27744-27753 (2007)

30. Castleberry, C.M., Limbach, P.A.: Relative quantitation of transfer RNAs using liquid chromatography mass spectrometry and signature digestion products. Nucleic Acids Res. 38, e162 (2010)

31. Juhling, F., Morl, M., Hartmann, R.K., Sprinzl, M., Stadler, P.F., Putz, J.: tRNAdb 2009: Compilation of tRNA sequences and tRNA genes. Nucleic Acids Res. 2009(37), D159-D162 (2009)

32. Sprinzl, M., Hartmann, T., Meissner, F., Moll, J., Vorderwulbeckbecke, T.: Compilation of tRNA sequences and sequences of tRNA genes. Nucleic Acids Res. 15, r53-r188 (1987)

33. Davis, D.R.: Biophysical and conformational properties of modified nucleosides in RNA (nuclear magnetic resonance studies). In: Grosjean, H., Benne, R. (eds.) Modification and editing of RNA, pp. 103-112. ASM Press, Washington, DC (1998)

34. Quigley, G.J., Wang, A.H.J., Seeman, N.C.: Hydrogen bonding in yeast phenylalanine transfer RNA. Proc. Natl. Acad. Sci. U.S.A. 72, 48664870 (1975)

35. Wahab, S., Rowley, K., Holmes, W.: Effects of tRNA ${ }^{\text {Leu I }}$ overproduction in Escherichia coli. Mol Microbiol. 7, 253-263 (1993) 